\title{
Quantitative analysis of turbostratically disordered nontronite with a supercell model calibrated by the PONKCS method
}

\author{
Xiaodong Wang, Robert D. Hart, Jian Li, Robbie G. McDonald and Arie \\ van Riessen
}

J. Appl. Cryst. (2012). 45, 1295-1302

Copyright (C) International Union of Crystallography

Author(s) of this paper may load this reprint on their own web site or institutional repository provided that this cover page is retained. Republication of this article or its storage in electronic databases other than as specified above is not permitted without prior permission in writing from the IUCr.

For further information see http://journals.iucr.org/services/authorrights.html

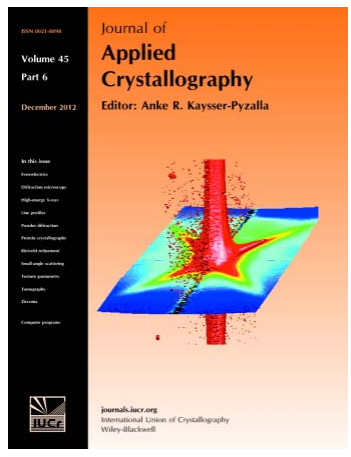

Journal of Applied Crystallography covers a wide range of crystallographic topics from the viewpoints of both techniques and theory. The journal presents papers on the application of crystallographic techniques and on the related apparatus and computer software. For many years, the Journal of Applied Crystallography has been the main vehicle for the publication of small-angle scattering papers and powder diffraction techniques. The journal is the primary place where crystallographic computer program information is published.

Crystallography Journals Online is available from journals.iucr.org 
Journal of

Applied

Crystallography

ISSN 0021-8898

Received 30 June 2012

Accepted 25 September 2012

(C) 2012 International Union of Crystallography

Printed in Singapore - all rights reserved

\section{Quantitative analysis of turbostratically disordered nontronite with a supercell model calibrated by the PONKCS method}

\author{
Xiaodong Wang, ${ }^{\text {a* }}$ Robert D. Hart, ${ }^{\text {a }}$ Jian Li, ${ }^{\mathrm{b}}$ Robbie G. McDonald ${ }^{\mathrm{b}}$ and Arie van \\ Riessen $^{\mathrm{a}}$ \\ ${ }^{a}$ Centre for Materials Research, Curtin University, GPO Box U1987, Perth, WA 6845, Australia, and \\ ${ }^{\mathbf{b}}$ Division of Process Science and Engineering, CSIRO, PO Box 7229, Karawara, WA 6152, \\ Australia. Correspondence e-mail: tony.wang@curtin.edu.au
}

Two calibration-based quantitative X-ray diffraction (XRD) models for turbostratically disordered Bulong nontronite, the PONKCS (partial or no known crystal structure) approach and the supercell structural model, were compared in terms of the accuracy and refinement error from Rietveld quantitative phase analysis. The PONKCS approach achieved improved nontronite quantitative results with synchrotron diffraction patterns compared with those achieved with laboratory XRD data as a result of better data quality and the use of Debye-Scherrer geometry with significantly reduced preferred orientation effects. The introduction of a peak shape modifier (spherical harmonics) to correct the quantification result is mainly useful for laboratory XRD patterns containing nontronite collected from Bragg-Brentano geometry with appreciable preferred orientation effects. A novel calibration approach for the nontronite supercell model was developed, based on the Rietveld quantitative formula in the TOPAS symbolic computation system. The calibrated supercell model achieved better accuracy (deviation within $1 \mathrm{wt} \%$ ) and lower refinement error than the PONKCS approach because the physically based description of turbostratic disorder requires fewer refinable parameters than the PONKCS approach. The drawbacks and limitations of the supercell approach are also discussed.

\section{Introduction}

High-grade sulfide nickel resources are declining. In contrast, there are vast resources of low-grade $(<0.8 \mathrm{wt} \% \mathrm{Ni})$ nickel laterites in Western Australia that are currently uneconomic to process (Dalvi et al., 2004). Heap leaching has advantages of much lower energy usage and capital requirements compared to high-pressure acid leaching, but a major problem is that the leaching behaviour of the ores is often unpredictable. Some ores leach well while other superficially similar ores are acid resistant and leach slowly with variable recoveries. The differences between these ores are not well characterized or understood.

The investigation of the influences of mineralogy on leaching, the prediction of leaching behaviour and improvements in leaching technology require accurate quantitative phase analysis, especially for nontronite, which is a major nickel-containing phase. The nontronite phase has been the most difficult to characterize, with a substantial fraction often reported as unaccounted or 'amorphous' content in X-ray diffraction studies. No suitable structural model is available for nontronite because this clay mineral is always turbostratically disordered, showing asymmetric nonbasal diffraction profiles. The PONKCS method (partial or no known crystal structure; Scarlett \& Madsen, 2006), which is particularly useful for quantification of disordered materials, was improved by using spherical harmonics as a peak shape modifier and applied to the quantitative analysis of nontronite from Western Australia to achieve an absolute quantification error of less than $5 \mathrm{wt} \%$ using laboratory X-ray diffraction (XRD) data (Wang et al., 2011). However, the model is not physically based and still demonstrates substantial errors at low nontronite concentrations. The accuracy and Rietveld refinement error of the modified PONKCS approach with synchrotron XRD patterns are here compared with laboratory XRD analysis.

A supercell model previously developed by Ufer et al. (2004) using the BGMN program (Bergmann et al., 2010) was reported to be suitable for quantitative analysis of smectite in bentonites; this approach involved the measurement of the cation exchange capacity (CEC) for interlayer cations using $\mathrm{Cu}$ triethylenetetramine (Ufer et al., 2008). The supercell structure was modified and incorporated into the TOPAS (Total Pattern Analysis Solutions; Bruker, 2009) software and achieved good fitting of the asymmetric nonbasal diffraction peaks (Wang et al., 2011). However, this model requires calibration in order to be used successfully for nontronite quan- 
Table 1

Quantitative results of six synthetic mixtures of goethite (Goe), quartz (Qtz) and nontronite (Nont).

Figures in brackets represent the $1 \sigma$ uncertainty on the last decimal place.

\begin{tabular}{|c|c|c|c|c|c|c|c|c|c|}
\hline \multirow[b]{2}{*}{ Sample ID } & \multicolumn{3}{|c|}{ Weighed wt $\%$ of synthetic laterites } & \multicolumn{3}{|c|}{ Calculated wt \% by SH-assisted PONKCS model } & \multicolumn{3}{|c|}{ Calculated wt $\%$ by calibrated supercell model } \\
\hline & Nont & Goe & Qtz & Nont & Goe & Qtz & Nont & Goe & Qtz \\
\hline NGQ1 & 10.03 & 70.13 & 19.84 & $6.1(3)$ & $72.8(2)$ & $21.12(8)$ & $9.3(3)$ & $70.0(2)$ & $20.78(10)$ \\
\hline NGQ2 & 15.07 & 55.02 & 29.91 & $14.3(2)$ & $55.06(15)$ & $30.66(10)$ & $14.2(2)$ & $55.17(17)$ & 30.59 (11) \\
\hline NGQ3 & 19.96 & 39.96 & 40.08 & $19.40(17)$ & $39.76(9)$ & $40.84(10)$ & $19.71(19)$ & $39.84(11)$ & $40.45(11)$ \\
\hline NGQ4 & 30.02 & 20.08 & 49.90 & $30.15(15)$ & $19.58(6)$ & $50.27(12)$ & 30.09 (17) & $20.20(7)$ & $49.71(13)$ \\
\hline NGQ5 & 34.97 & 50.01 & 15.02 & 33.30 (18) & $50.76(14)$ & $15.94(6)$ & $35.06(18)$ & $49.44(14)$ & $15.50(7)$ \\
\hline NGQ6 & 49.97 & 24.92 & 25.11 & $50.35(10)$ & $24.54(6)$ & $25.11(6)$ & $50.36(15)$ & $24.94(9)$ & $24.70(10)$ \\
\hline
\end{tabular}

titative analysis. A method for calibrating the interlayer cation occupancies for the supercell model has been developed, resulting in significant improvement of the quantitative bias to within $1 \mathrm{wt} \%$. Both the supercell and PONKCS models have been applied to XRD data obtained from the powder diffraction beamline at the Australian Synchrotron, and the results demonstrate the advantages of using a physically based model to quantify turbostratically disordered clay minerals.

\section{Materials and methods}

\subsection{Clay separation}

Green nontronite-rich clay $(\sim 0.5 \mathrm{~kg})$ collected from the Bulong mine site, Western Australia, was crushed into powder ( $<1 \mathrm{~mm}$ ) using a Rocklabs model 1A ring mill. A sample of $100 \mathrm{~g}$ was transferred into a $500 \mathrm{ml}$ cylinder, soaked with deionized water and shaken vigorously. The cylinder was left standing until all the particles settled and the clear supernatant was decanted. The powder was washed with deionized water in this way several times until the solids started to form a gel (i.e. the particles do not settle under gravity because the total dissolved salt level of the liquid has decreased). This gel was further diluted with deionized water into $5 \times 500 \mathrm{ml}$ cylinders and allowed to settle under gravity for $4 \mathrm{~h}$, at which point the green supernatant suspensions were collected in a large beaker and dried at $343 \mathrm{~K}$. After the water had evaporated the residual solid remained as hard green aggregates, which were crushed with an agate mortar and pestle until the powder passed through a 100 mesh sieve.

A nontronite standard, NAu-1, from Port Lincoln, South Australia (Keeling et al., 2000), was used as a control for chemical assay. The NAu-1 nontronite standard was received as bright-green hard lumps. Dark stains indicating manganese oxide impurities were carefully removed using a scalpel, and the nontronite crumbs were hand milled with an agate mortar and pestle and passed through a 100 mesh sieve.

The enriched Bulong and NAu-1 nontronite powders were further purified to remove kaolinite using high-speed centrifugation. Five gram sub-samples of each nontronite were agitated in $1 \mathrm{l}$ of $0.02 \mathrm{M} \mathrm{LiCl}$ solution using rolling plastic bottles for $48 \mathrm{~h}$ to lithium saturate the nontronite. The resulting suspensions were centrifuged (Heraeus Multifuge $3 \mathrm{~S}-\mathrm{R}$ centrifuge) to separate the clays from the exchanged $\mathrm{LiCl}$ solutions, which were collected and referred to as the 'first cation exchange liquor'. The centrifuged clays were treated with $0.02 \mathrm{M} \mathrm{LiCl}$ solution again to ensure that the resulting clays were mostly $\mathrm{Li}$ saturated. Milli-Q water was used to wash and disperse the Li-saturated clays, which were then repeatedly centrifuged in order to collect the supernatant suspensions containing fine clay fractions of less than $0.2 \mu \mathrm{m}$. The suspensions were collected in large beakers then flocculated with excess $\mathrm{CaCl}_{2}$ and left to settle overnight before the clear supernatants were decanted. Residual $\mathrm{CaCl}_{2}$ solution was removed by centrifugation and $\mathrm{Ca}^{2+}$ saturation was repeated. Excess $\mathrm{CaCl}_{2}$ was removed by repeated dispersion of the clay in Milli-Q water followed by centrifugation until the supernatant become turbid. The settled clays were collected and oven dried at $343 \mathrm{~K}$. The dried solids were hand milled with an agate mortar and pestle to pass through a 100 mesh sieve and are referred to as 'purified Bulong nontronite' and 'purified NAu-1 nontronite', respectively.

\subsection{Inductively coupled plasma emission spectrometry}

The chemical compositions of the first cation exchange liquors were determined by inductively coupled plasma emission spectrometry (ICP-OES) to estimate the species and amounts of interlayer cations for both Bulong nontronite and the NAu-1 standard. Both purified Ca-saturated nontronite powders were also analysed by ICP-OES after hightemperature fusion with Sigma Chemicals 12:22 lithium borate flux and dissolution in water.

\subsection{Bulong nontronite chemical formula}

The approach adopted for calculation of the Bulong nontronite structural formula was the same as that reported for NAu-1 (Keeling et al., 2000). The equations for determining the 2:1 phyllosilicate chemical formula as described in CLAYFORM (Bodine, 1987) were rewritten in Microsoft Excel. The chemical formula of Bulong nontronite was generated based on 22 equivalent $\mathrm{O}$ atoms using the ICP assay of purified Ca-saturated Bulong nontronite.

\subsection{Standard mixtures and synthetic laterites}

In order to calibrate both the PONKCS and supercell models, the enriched Bulong nontronite was mixed with 10, 30 and $50 \mathrm{wt} \%$ fluorite $\left(\mathrm{CaF}_{2}\right.$, Sigma-Aldrich, $>99.5 \%$, powder $\sim 325 \mathrm{mesh}$ ) using a McCrone micronizing mill for $5 \mathrm{~min}$ to prepare three standard mixtures. Fluorite was chosen because its mass absorption coefficient is close to that of nontronite at 
the $1 \AA \mathrm{X}$-ray wavelength used in the synchrotron experiment. Laboratory-grade ethanol $(10 \mathrm{ml})$ was chosen to assist milling and corundum pellets were used as the milling medium. The milled slurries were oven dried at $343 \mathrm{~K}$ and gently ground using an agate mortar and pestle. Six independent synthetic limonitic laterites were prepared by mixing Bulong nontronite with goethite (Synergy Pigments Australia Pty Ltd) and quartz (pulverized cleaning sand from Cook Industrial Minerals Pty Ltd), to simulate various common phase compositions of Western Australian nickel laterite (Elias et al., 1981; Landers et al., 2009; Singh \& Gilkes, 1992). The pulverized quartz used in this study was not subject to size fraction separation and was hence suspected to be a polydisperse size system, i.e. having wide particle size distribution. Details of the weight ratios are shown in Table 1. The mixing method was the same as used in the standard mixture preparation, with $10 \mathrm{wt} \% \mathrm{CaF}_{2}$ as an internal standard. Quartz and goethite were verified to be pure phases and lattice parameters were determined with $2 \theta$ correction (zero error and sample displacement error) by an internal standard method (McCusker et al., 1999).

\subsection{Synchrotron X-ray diffraction}

The purified Bulong and NAu-1 nontronite samples, three standard mixtures, and six synthetic laterites were loaded into $0.3 \mathrm{~mm}$ internal diameter borosilicate capillaries (GLAS, Schönwalde, Germany) for synchrotron diffraction measurements. The capillaries were agitated in an ultrasonic bath to assist powder loading and promote random orientation of the particles.

Synchrotron X-ray powder diffraction patterns of all samples were collected on the Powder Diffraction Beamline (10-BM-1) at the Australian Synchrotron using a Mythen microstrip position-sensitive detector and Debye-Scherrer geometry (Schmitt et al., 2003). The wavelength was set to $1 \AA$ and measured accurately to be $0.999 \AA$ from NIST Standard Reference Material 660 $\mathrm{LaB}_{6}$ diluted with diamond powder. This standard was also used to obtain the instrumental profile of the beamline under the experimental settings employed. The Mythen detector has small gaps between the 16 detector modules so two patterns were collected with a small $2 \theta$ angle offset $\left(0.5^{\circ}\right)$. Each of these patterns was collected for $450 \mathrm{~s}$ and spliced using the DataPro (Version 2.7 beta; Gu, 2011) program, accounting for decay of the beam intensity via the ion chamber counts. The combined patterns ranged from 1.2 to $81^{\circ} 2 \theta$ covering $d$ spacings from 40 to $0.77 \AA$, respectively. The sample capillaries were spun at $15 \mathrm{rmin}^{-1}$ during data collection. The spinner incorporates a motorized goniometer head with image capture and processing software designed to automatically align capillary eucentric positions reproducibly to $\pm 10 \mu \mathrm{m}$.

\subsection{Applying the PONKCS method with synchrotron data}

The accuracy of the PONKCS quantification method was compared for Bulong nontronite XRD patterns collected at the Australian Synchrotron using Debye-Scherrer geometry and laboratory data collected using Bragg-Brentano geometry in our previous study (Wang et al., 2011). Calcite was included in the Pawley fitting of synchrotron data of purified Ca-saturated Bulong nontronite as residual calcium chloride appears to have transformed to calcite during oven drying. The approach used to build a lattice model (hkI_Is) for Bulong nontronite is similar to that described previously (Wang et al., 2011) but is briefly described here.

The instrumental convolutions, zero error and wavelength of synchrotron radiation were determined from the NIST $660 \mathrm{~b}$ $\mathrm{LaB}_{6}$ standard. The capillary background was modelled from the pattern of an empty capillary using a peaks phase group, which is essentially a group of peaks (xo_Is) with the peak shape modifier described individually and scale factors linked and refinable (Wang et al., 2011). A zero-order Chebyshev background was used to minimize the correlation between the Chebyshev background and nontronite Bragg humps. A background function fit_obj $=a /(X-b)(a, b$ refinable $)$ was used to fit the low-angle high background prior to the nontronite 001 basal reflection; the fit was much closer than that achieved by the One_on_X macro, which uses the basic inverse proportion function fit_obj $=a / X$.

The $C 2 / m$ space group was selected for the nontronite lattice model (hkl_Is) used in the PONKCS method on the basis of previous work (Wang et al., 2011). The unit-cell $b$ value can be predetermined from the 060 diffraction peak position [1.5160 (1) $\AA$ ] by fitting it to a peaks phase component (d_Is). Pawley fitting was then used to extract reflections (hkls), the number of which was gradually reduced by deleting zero- and low-intensity reflections and those reflections with intensity less than the corresponding error until the fitting parameter $R_{\text {wp }}$ (weighted profile $R$ factor) began to increase. Spherical harmonics (SH) were used to reproduce asymmetric peak shapes, anisotropic strain and size broadening in this process. The final model was achieved by refining all parameters to achieve the best fit to the measured Bulong nontronite pattern. The reflection intensities and SH coefficients were then fixed. The $Z M$ (unit-cell mass) factor of the nontronite lattice model was calibrated using the synchrotron XRD patterns of the nontronite and $\mathrm{CaF}_{2}$ standard mixtures described in $\$ 2.4$.

\subsection{Calibration of the supercell model by the PONKCS method}

For the supercell model the instrumental convolutions, zero error, radiation wavelength and background treatment were the same as those used in §2.6. The species and occupancies of octahedral and tetrahedral cations in the nontronite supercell model were determined from the Bulong nontronite formula calculated in $\$ 2.3$. The atom coordinates were adopted from the $c i s$-vacant nontronite structure model, solved by Tsipursky \& Drits (1984), as an approximate structure. The rigid-body rotation and translation were revised from the original supercell model (http://www.bgmn.de/download-structures. html) by linking them together with subcell parameters (see Appendix $A^{\mathbf{1}}$ ). This guarantees that the double-layer hydrated

\footnotetext{
${ }^{1}$ Appendix $A$ (containing a TOPAS input file) is available as supplementary material from the IUCr electronic archives (Reference: NB5033). Services for accessing this material are described at the back of the journal.
} 
interlayer cations are always placed at the centre of the subcell with two of the $\mathrm{H}_{2} \mathrm{O}$ planes parallel to the $a b$ plane, regardless of subcell deformation during refinement, as shown in Fig. 1. The occupancy of the interlayer cations was initially based on the molar ratio of major exchangeable cations from the ICP assay of the first cation exchange liquor. The unit-cell $b$ value was predetermined from the 060 diffraction peak position. This model was built entirely on the basis of the ICP chemical assay and is referred to as the 'uncalibrated supercell model'.

The supercell model calibration involves two refinement steps. A normal refinement with the uncalibrated nontronite supercell model and the structural model of $\mathrm{CaF}_{2}$ was performed first, in order to achieve a best fit to the patterns of

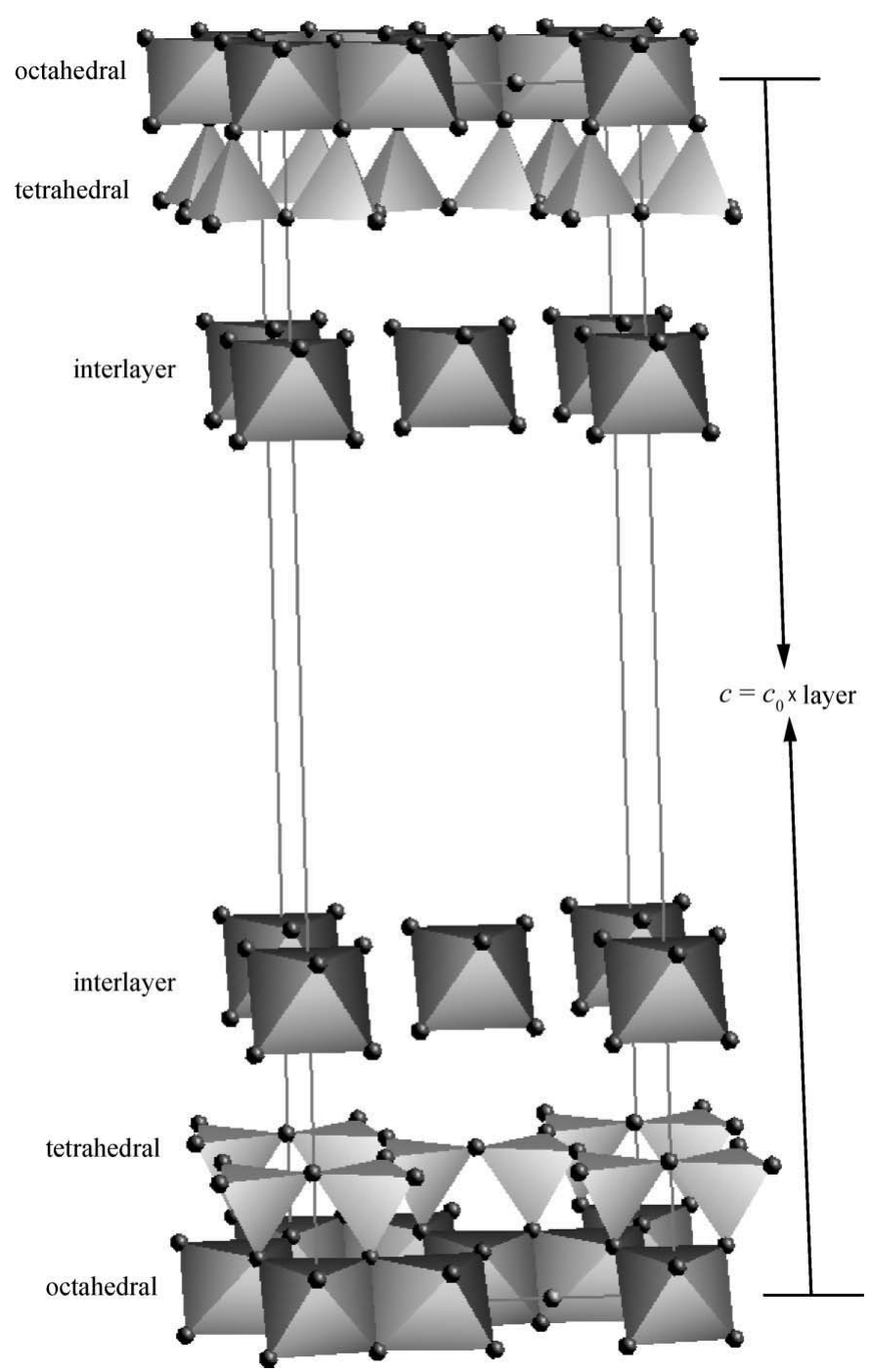

Figure 1

The nontronite supercell model used in this study drawn using the $T O P A S$ rigid-body editor, indicating the interlayer hydrated cations were calculated twice in the unit cell. The $\mathbf{c}$ direction length of the supercell ' $c$ ' is 'layer' times longer than that of subcell ' $c_{0}$ '. To save space, the case where layer $=2$ is shown, though a layer $=9$ model was used in this study. The rigid-body rotations and translations given in Appendix $A$ were linked to unit-cell parameters to ensure that the double-layer hydrated cations were always placed at the centre of the subcell (layer $=1$ ) with two of the $\mathrm{H}_{2} \mathrm{O}$ planes parallel to the $a b$ plane, while the unit-cell dimensions were refined. the standard mixtures prepared in $\$ 2.4$. The scale factors and lattice parameters of the internal standard, $\mathrm{CaF}_{2}$ in this case, were determined from this refinement. These parameters of the internal standard were then fixed for a 'second refinement', in which the Rietveld quantification formula was used in a reverse manner. In contrast to measuring the interlayer cation occupancy with the CEC method (Ufer et al., 2008), the weighed percentages of components in standard mixtures were used to refine the lattice parameters and a coefficient for the nontronite interlayer cation occupancies, as shown in Fig. 2. As will be discussed in $\$ 3.3 .1$, the starting value of this coefficient for the nontronite interlayer cation occupancies is 0.5 . This coefficient and the lattice parameters of Bulong nontronite used in the final Bulong nontronite supercell model were determined by arithmetically averaging their refined values from the 'second refinement' for all three standard mixtures. These parameters were fixed in the final supercell model used for the quantitative analysis of nontronite in the synthetic laterite mixtures.

\section{Results and discussions}

\subsection{Bulong nontronite formula}

The ICP assays for the first cation exchange liquor and the purified $\mathrm{Ca}$-saturated Bulong and NAu-1 nontronites are shown in Table 2. The exchangeable cations for the two nontronites are quite different. Calcium is the dominant interlayer exchangeable cation for NAu-1, while for Bulong nontronite it is sodium. The ICP assay for the purified NAu-1 sample matches that previously reported (Keeling et al., 2000). The ICP analysis of the purified Bulong nontronite confirms that $\mathrm{Ni}$ is not present as an exchangeable cation but is fixed

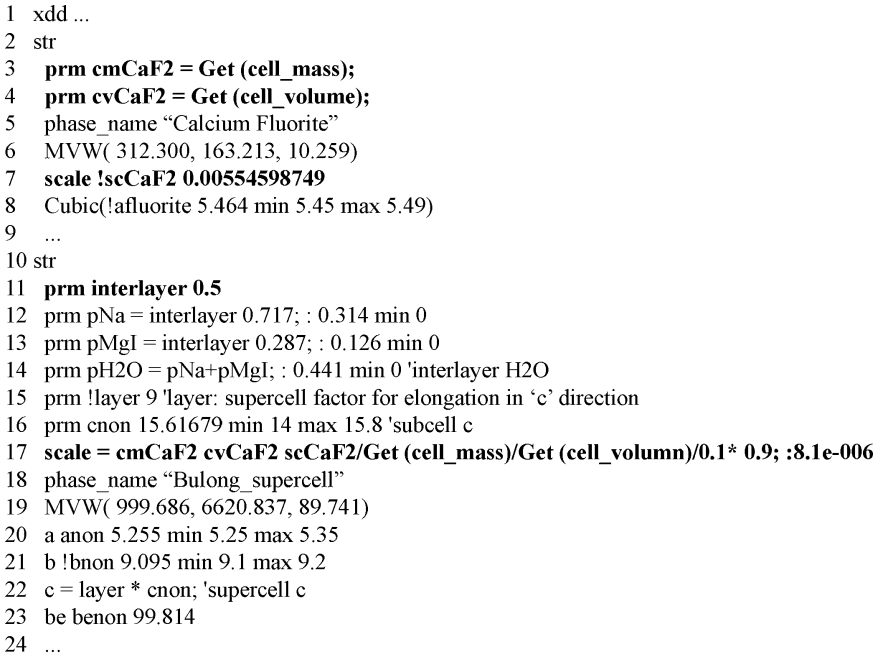

Figure 2

A section of the TOPAS input file showing reversal of the Rietveld quantification formula. In line 17, the nontronite scale factor is linked with the unit-cell mass and volume by refining the interlayer occupancy coefficient and lattice parameters. The values of ' 0.1 ' and ' 0.9 ' used in line 17 are the weight percentages of $\mathrm{CaF}_{2}$ and nontronite in this standard mixture, respectively. 
Table 2

ICP assays for the 'first cation exchange liquors' and the purified Ca-saturated Bulong and NAu-1 nontronites.

\begin{tabular}{|c|c|c|c|c|c|c|c|c|c|c|c|c|c|}
\hline \multirow[b]{2}{*}{ Sample } & & & & \multicolumn{10}{|c|}{ Exchangeable cation $\left(\mathrm{mg} \mathrm{g}^{-1}\right)$} \\
\hline & & & & Co & $\mathrm{Fe}$ & $\mathrm{Ni}$ & $\mathrm{Mg}$ & $\mathrm{Al}$ & $\mathrm{Si}$ & $\mathrm{Na}$ & $\mathrm{Ca}$ & $\mathrm{K}$ & $\mathrm{Cr}$ \\
\hline \multirow{2}{*}{\multicolumn{2}{|c|}{ First cation exchange liquors }} & \multirow{2}{*}{\multicolumn{2}{|c|}{$\begin{array}{l}\text { NAu-1 } \\
\text { Bulong Nontronite }\end{array}$}} & $<0.04$ & $<0.04$ & $<0.04$ & 1.03 & $<0.04$ & 0.58 & 1.12 & 3.02 & $<0.04$ & $<0.04$ \\
\hline & & & & $<0.04$ & $<0.04$ & $<0.04$ & 1.94 & $<0.04$ & 0.30 & 4.58 & 0.10 & $<0.04$ & $<0.04$ \\
\hline & & & \multicolumn{11}{|c|}{ Elemental concentration (\%) } \\
\hline \multicolumn{3}{|l|}{ Sample } & $\mathrm{CoO}$ & $\mathrm{Fe}_{2} \mathrm{O}_{3}$ & $\mathrm{NiO}$ & $\mathrm{MgO}$ & \multicolumn{2}{|l|}{$\mathrm{Al}_{2} \mathrm{O}_{3}$} & \multicolumn{2}{|r|}{$\mathrm{Na}_{2} \mathrm{O}$} & $\mathrm{CaO}$ & $\mathrm{K}_{2} \mathrm{O}$ & $\mathrm{Cr}_{2} \mathrm{O}_{3}$ \\
\hline \multirow[t]{2}{*}{ Purified powders } & NAu-1 & & - & 36.0 & 0.022 & 0.270 & \multirow{2}{*}{$\begin{array}{l}8.02 \\
10.57\end{array}$} & & \multirow{2}{*}{\multicolumn{2}{|c|}{$\begin{array}{l}0.020 \\
0.107\end{array}$}} & 4.72 & 0.091 & 0.019 \\
\hline & Bulong & ontronite & 0.060 & 24.4 & 2.52 & 1.361 & & & & & 4.47 & 0.099 & 0.849 \\
\hline
\end{tabular}

within the octahedral layer. The structural formula of Bulong nontronite calculated as described in $\$ 2.3$ is $\mathrm{M}_{1.29}{ }^{+}-$ $\left[\mathrm{Si}_{7.33}{ }^{4+} \mathrm{Al}_{0.67}{ }^{3+}\right]\left[\mathrm{Al}_{0.95}{ }^{3+} \mathrm{Fe}_{2.40}{ }^{3+} \mathrm{Ni}_{0.26}{ }^{2+} \mathrm{Co}_{0.01}{ }^{2+} \mathrm{Mg}_{0.26}{ }^{2+} \mathrm{Cr}_{0.09}{ }^{3+}\right]-$ $\mathrm{O}_{20}(\mathrm{OH})_{4}$, where $M^{+}$represents monovalent cations.

\subsection{Re-evaluation of the PONKCS model with synchrotron data}

The accuracy and Rietveld refinement error of the PONKCS approach with synchrotron XRD patterns collected using Debye-Scherrer geometry were compared with the previous analysis of laboratory XRD data collected using Bragg-Brentano geometry (Wang et al., 2011). The fitting and bias of the quantitative results from the weighed percentages for the synthetic laterites based on the original PONKCS method (Scarlett \& Madsen, 2006) are shown in Figs. 3 and 4, respectively. The nontronite 001 basal diffraction peaks were fitted well but the asymmetric nonbasal diffraction bands were poorly fitted (Fig. 3). However, quantitative results for the synthetic laterite samples (Fig. $4 b$ ) show better accuracy with lower refinement error than those obtained from laboratory XRD data using the same method (Fig. 4a). The much lower refinement error and standard uncertainty of the phase weight percentages can be partially attributed to the superior signalto-noise ratio of the synchrotron radiation data (Toby, 2006).

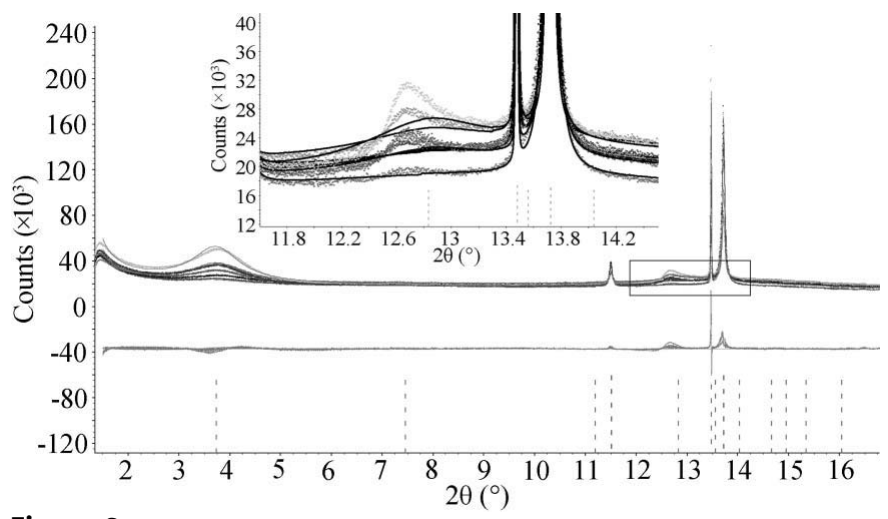

Figure 3

Simultaneous fitting of synchrotron patterns of synthetic laterite ores with overall goodness of fit $(\mathrm{GOF})=3.541, R_{\mathrm{wp}}=4.008$ and $R_{\exp }=1.132$. The asymmetric nonbasal diffraction bands were poorly fitted. Inset: enlargement of the nontronite asymmetric nonbasal diffraction from 11.6 to $14.5^{\circ} 2 \theta$.
The fitting and the quantification results of the synthetic laterite samples calculated using spherical harmonics to model peak shape (modified PONKCS approach) are presented in Fig. 5 and Table 1. The biases of quantitative results from the weighed percentages for the synthetic laterites are shown in Fig. 6. The fit of the nontronite asymmetric nonbasal diffraction bands with the modified PONKCS method (Fig. 5) is significantly better than the fitting generated by the original PONKCS method (Fig. 3). The quantification results (Fig. 6b) are again more accurate, with lower refinement error than those obtained from the laboratory data using the same method (Fig. 6a). However, the synchrotron-based results with

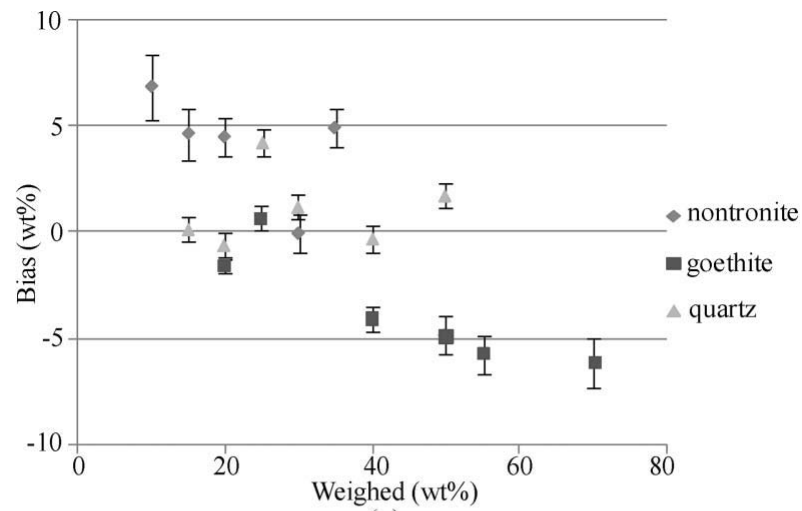

(a)

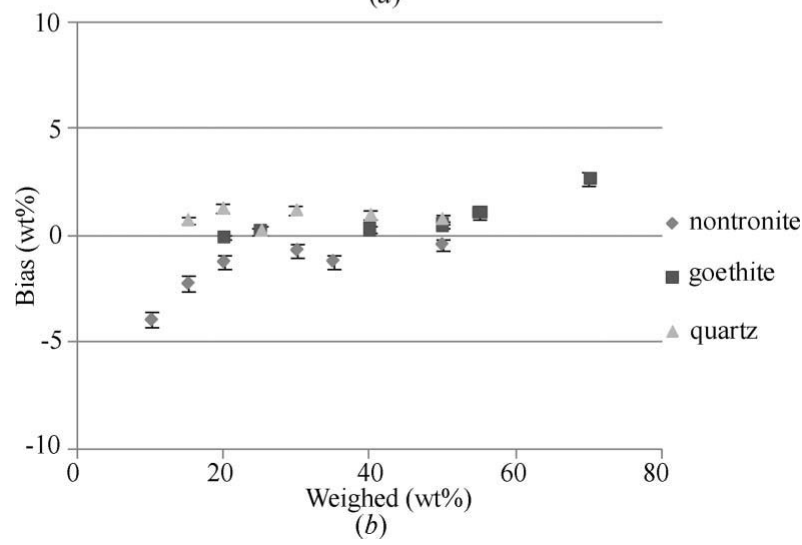

Figure 4

Difference plot showing the weighed percentage and quantitative analysis results of synthetic laterite ores from the original PONKCS method using (a) laboratory data (Wang et al., 2011) and (b) synchrotron data. 
the lowest nontronite content (10 wt \%) have a large bias. The underestimation of nontronite content is due to the low 001 diffraction intensities correlating with the capillary background, driving the nontronite scale factor to a lower value. This is confirmed by the least-squares covariance matrix from the refinement.

The quantitative results derived by the modified PONKCS method for synchrotron data (Fig. 6b) did not show obvious

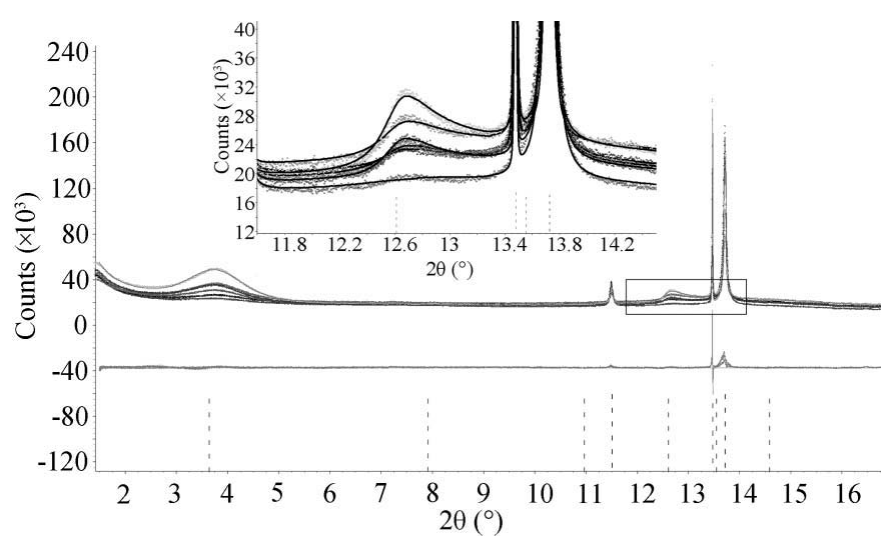

Figure 5

Simultaneous fitting of synchrotron patterns of synthetic laterite ores with overall GOF $=3.301, R_{\mathrm{wp}}=3.735$ and $R_{\mathrm{exp}}=1.132$. The asymmetric nonbasal diffraction bands were well fitted. Inset: enlargement of the nontronite asymmetric nonbasal diffraction from 11.6 to $14.5^{\circ} 2 \theta$.

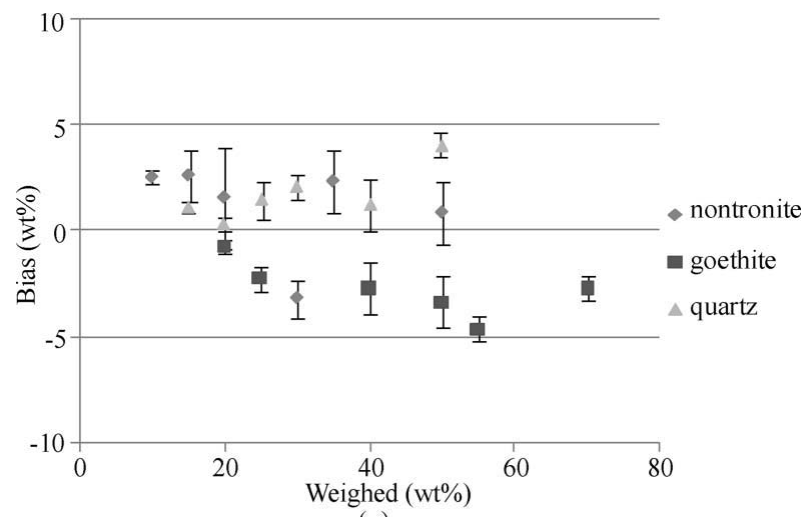

(a)

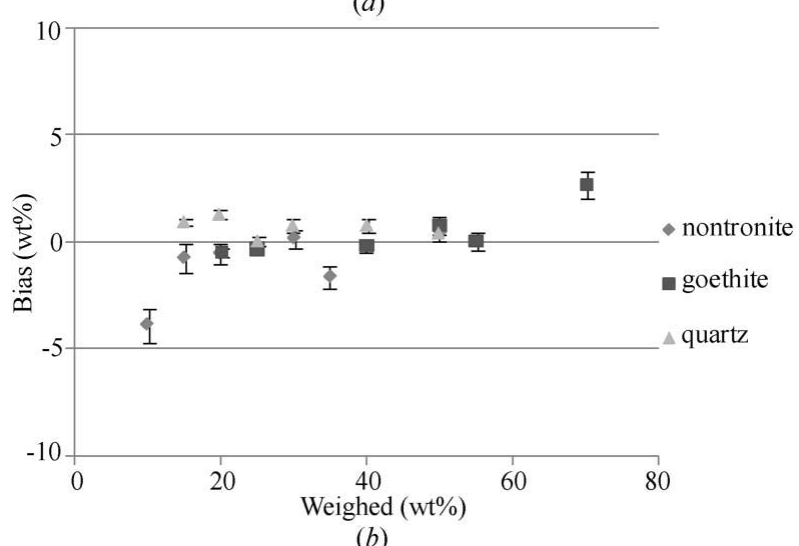

(b)

Figure 6

Difference plot showing the weighed percentage and quantitative analysis results of synthetic laterite ores from the modified PONKCS method using (a) laboratory data (Wang et al., 2011) and (b) synchrotron data. improvement from those derived by the original PONKCS method (Fig. 4b). However, the improvement of the quantitative results due to the use of spherical harmonics to model peak shape is apparent for laboratory XRD data obtained using Bragg-Brentano geometry (Wang et al., 2011). This is due to the effect of sample preferred orientation, which is unavoidable for front-pressed samples measured with BraggBrentano geometry but can be alleviated using DebyeScherrer geometry. Correct modelling of the nontronite nonbasal diffraction bands means that the quantitative results are less correlated with the intensity of the major 001 diffraction peak. When the nonbasal diffraction bands are not fitted correctly, the nontronite scale factor, which directly links to the phase percentage, is highly susceptible to preferred orientation effects associated with flat-plate sample preparation (Kleeberg et al., 2008). Hence a significant improvement in quantitative accuracy is found by fitting the nonbasal diffraction bands obtained in laboratory Bragg-Brentano geometry, but this is not the case in synchrotron DebyeScherrer geometry where preferred orientation effects are negligible. Fitting nonbasal diffraction peaks could also assist in determining other phases with overlapping peaks in the same $2 \theta$ angle range. The $R_{\mathrm{wp}}$ factor decreased from 4.008 to 3.735 when spherical harmonics were used to define the peak shape. This is not a large $R_{\mathrm{wp}}$ decrease because the misfit of the main diffraction peak of quartz appears to be the major contributor to $R_{\mathrm{wp}}$ (Fig. 5). Particle size analysis of scanning electron microscopy images of the quartz powder used in synthetic mixtures suggests it has a polydisperse size distribution and therefore the normal 'double Voigt' approach could not reproduce the peak profile (Langford et al., 2000; David et al., 2010).

\subsection{Quantitative analysis using the supercell model approach}

3.3.1. Uncalibrated supercell model. An uncalibrated supercell model (atom occupancies solely determined from chemical assay) usually gives rise to inaccurate quantitative results, although good fitting for a nontronite pattern can be achieved, as shown in Fig. 7. This may be a result of the error associated with the chemical assay, or the deviation between the real atom positions of Bulong nontronite and those reported by Tsipursky \& Drits (1984). The uncalibrated supercell model resulted in quantitative discrepancies of more than $5 \mathrm{wt} \%$ for the synthetic laterite samples, as shown in Fig. 8(a).

According to the Rietveld quantitative theory the $Z M$ factor for this supercell model should be calibrated to achieve accurate quantitative analysis of nontronite in synthetic laterites. However, unlike the peaks phase (xo_Is) or Pawley phase (hkl_Is) reported previously (Wang et al., 2011), the $Z M$ factor of a structural model (str) cannot be manually assigned. It solely depends on the atomic species and their occupancies in the unit cell. The atomic species determined by ICP assay should be more reliable than their occupancies as calculated for the nontronite chemical formula. The variation of the octahedral and tetrahedral cation occupancies from 
chemical assay errors would not affect the $Z M$ factor substantially since the stoichiometric numbers for these two sites are fixed to 4 and 8 , respectively (if the octahedral vacancy is not considered), i.e. a decrease in aluminium would be compensated by an increase in iron, using the CLAYFORM algorithm. On the other hand, the variation of inter-

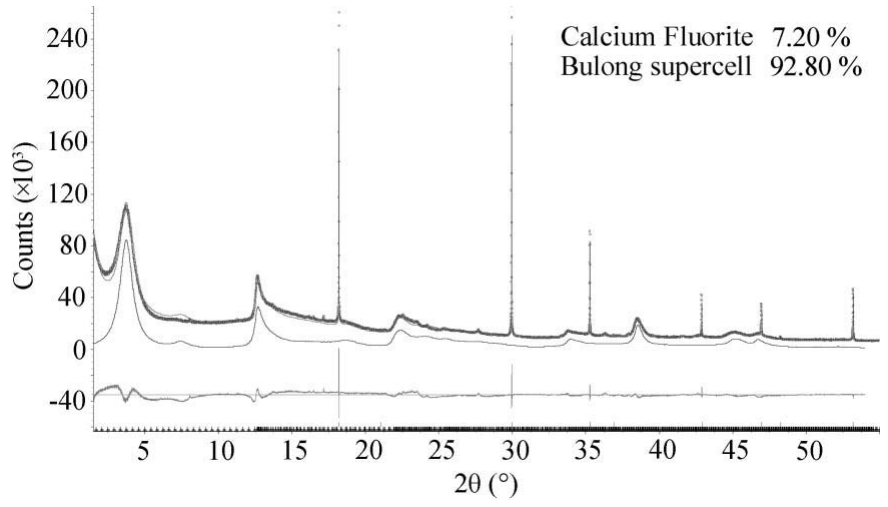

Figure 7

Fitting of the asymmetric nonbasal diffraction band using the uncalibrated supercell model. The quantitative results show an overestimation for the Bulong nontronite in this 9:1 mixture with $\mathrm{CaF}_{2}$. The reflection markers at the bottom indicate the significant number of additional diffraction peaks included in the calculation for each refinement step, making the calculation time consuming. The nontronite curve is highlighted.

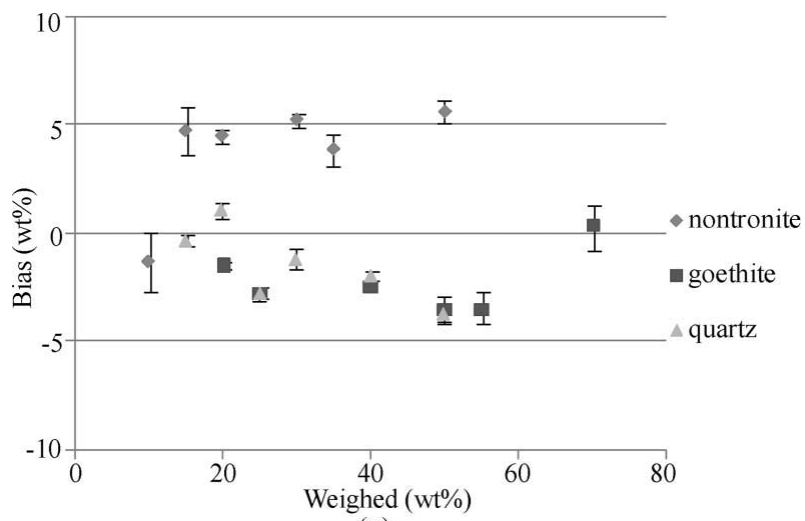

(a)

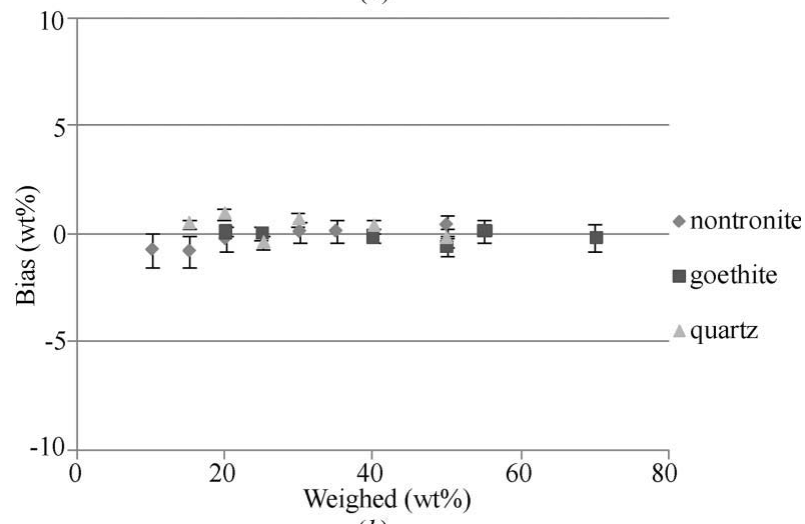

(b)

Figure 8

Difference plot showing the weighed percentage and quantitative analysis results of synthetic laterite ores using $(a)$ the uncalibrated supercell model and $(b)$ the calibrated supercell model. layer cation occupancies influences the unit-cell mass significantly. This is further amplified by the fact that the interlayer atoms are effectively calculated twice in the supercell approach, as shown in Fig. 1. Therefore, the coefficient of the interlayer cation occupancies should be calibrated with a starting value of 0.5 .

3.3.2. Calibrated supercell model. The calibrated coefficient of the interlayer cation occupancies derived from the three standard mixtures was 0.398 (6), which corresponds to a $Z M$ factor of 980 atomic mass units in the supercell model for Bulong nontronite. The averaged lattice parameters of Bulong nontronite from three standard mixtures correspond to a unitcell volume of $6637 \AA^{3}$ in the final Bulong nontronite supercell model. The product of the calibrated unit-cell mass and volume plays the same role as the calibrated $Z M V$ factor in the PONKCS approach (Wang et al., 2011). The calibrated coefficient of the interlayer cation occupancies does not have a physical meaning but is simply a factor for adjusting the unitcell mass to achieve the correct weight percentage. This calibration constant also absorbs residual sample-related effects such as microabsorption (Madsen et al., 2011) and other systematic errors, such as structural deviation and residual preferred orientation.

The quantitative results for the synthetic laterite samples using the calibrated supercell model are shown in Table 1 . The use of this model reduced the bias from the known weighed values to within $1 \mathrm{wt} \%$ (Fig. $8 b$ ). This shows greater accuracy than the modified PONKCS approach (Fig. 6b). This result also demonstrates the advantage of using a crystallographic structure-based model for the quantitative analysis of turbostratically disordered clay minerals. Only four parameters relating to the size and strain broadening of the basal and nonbasal diffraction peaks were refined. In contrast, for the modified PONKCS approach 12 parameters are required for the use of spherical harmonics to reproduce anisotropic size/strain broadening and associated asymmetries (three second-order spherical harmonics in the $C 2 / m$ space group). The larger number of parameters refined in the PONKCS Pawley model may therefore lead to correlations that impact on the calculated scale factor, thus preventing accurate phase quantification.

The calibration method used here for the supercell model for nontronite quantitative analysis was derived from the core idea of the PONKCS approach, which uses the target material to calibrate the quantitative X-ray diffraction (QXRD) model. Hence both approaches should be regarded as calibrationbased methods. It should be noted that, although the interlayer cation occupancies in the nontronite supercell model were refined during the calibration, this approach is purely to facilitate quantitative analysis and should not be considered for structure solution. An approach similar to that used by Manceau et al. (1998) is recommended for determination of the nontronite crystal structure.

The supercell model requires significantly more effort than the PONKCS method. Building the supercell model requires chemical assay data for the purified nontronite to determine the substituted metal cations and their occupancies, while the 
PONKCS approach only requires the target phase to be enriched. The PONKCS method is useful for any phase with unknown or partially known crystal structure, while the supercell model approach is only applicable to the turbostratically disordered clay minerals. Another drawback of the supercell approach is that the refinement is time consuming since nine times more reflections are included in the calculation in each refinement step (for this case where layer $=9$ in the supercell structure shown in Appendix $A$ ). On the other hand, the diffraction peaks used to define the Pawley phase in the PONKCS approach can be selectively deleted, as stated in $\$ 2.6$, reducing the calculation time. If the QXRD accuracy of the PONKCS approach is acceptable then it is the most direct and effective method for mining and metallurgy industries to perform quantitative mineralogy, where large numbers of samples are analysed.

\section{Conclusions}

The modified PONKCS approach (Wang et al., 2011) was applied to synchrotron data for standard mixtures and synthetic laterites, generating improved accuracy and lower refinement error relative to laboratory-based XRD data for the same samples. The benefit of using spherical harmonics as a peak shape modifier to improve the quantitative analysis was not as obvious for synchrotron-based data as previously found for laboratory XRD data (Wang et al., 2011). This is primarily because of the preferred orientation of the nontronite, which is exacerbated in the flat-plate samples prepared for laboratory-based Bragg-Brentano geometry XRD. Better fitting of the asymmetric nonbasal diffraction bands causes the quantitative results to become less dependent upon the primary basal diffraction peak, the intensity of which is highly susceptible to preferred orientation.

The TOPAS symbolic computation system (Coelho et al., 2011) provided an ideal platform to develop a novel structural model calibration approach for the interlayer cation occupancies in the nontronite supercell model using the patterns of standard mixtures with known weight ratios. The new calibrated supercell model was able to achieve quantitative analysis of nontronite in synthetic laterites to within $1 \mathrm{wt} \%$ of the weighed values.

This supercell calibration approach is aimed at quantitative analysis and should not be regarded as providing a structure solution. Although improved quantitative results were obtained, the time-consuming refinement, the need to chemically assay the pure target phase and the limitation to handling turbostratically disordered clay minerals make the supercell model less attractive than the PONKCS approach for mining and metallurgy industries that demand rapid, and often just comparative, mineralogical data.
The PhD scholarship of XW is funded by an Endeavour Postgraduate Award from the Department of Education, Employment and Workplace Relations, Australian Government. The research was partially funded by the Parker Cooperative Research Centre. The authors are also grateful for the financial support of the Australian Government through the CSIRO Minerals Down Under Flagship. We thank Mr Mark Raven, CSIRO Land and Water, for providing nontronite standard NAu-1 and the clay purification procedure. The clay purification work was conducted in the Waterford laboratories of CSIRO Process Science and Engineering. The authors acknowledge the technical assistance provided by Dr Justin Kimpton at the Australian Synchrotron Powder Diffraction Beamline (10-BM-1) and the synchrotron grant AS113/PD/4130 for beam time and travel.

\section{References}

Bergmann, J., Kleeberg, R., Ufer, K. \& Friedel, P. (2010). BGMN. Version 5.1.8. Dresden, Germany.

Bodine, M. W. (1987). Comput. Geosci. 13, 77-88.

Bruker (2009). DIFFRAC $C^{\text {plus }}$ TOPAS 4.2 User Manual. Bruker AXS $\mathrm{GmbH}$, Karlsruhe, Germany.

Coelho, A. A., Evans, J., Evans, I., Kern, A. \& Parsons, S. (2011). Powder Diffr. 26, S22-S25.

Dalvi, A. D., Bacon, W. G. \& Osborne, R. C. (2004). PDAC 2004 International Convention, 7-10 March 2004, Toronto, Canada.

David, W. I. F., Leoni, M. \& Scardi, P. (2010). Mater. Sci. Forum, 651, 187-200.

Elias, M., Donaldson, M. J. \& Giorgetta, N. E. (1981). Econ. Geol. 76, $1775-1783$.

Gu, Q. (2011). DataPro. Version 2.7. Australian Synchrotron, Melbourne, Australia, http://www.synchrotron.org.au/images/stories/ beamline/powder/Datapro2.7.zip.

Keeling, J. L., Raven, M. D. \& Gates, W. P. (2000). Clays Clay Miner. 48, 537-548.

Kleeberg, R., Monecke, T. \& Hillier, S. (2008). Clays Clay Miner. 56, 404-415.

Landers, M., Gilkes, R. J. \& Wells, M. A. (2009). Clays Clay Miner. 57, 751-770.

Langford, J. I., Louër, D. \& Scardi, P. (2000). J. Appl. Cryst. 33, 964-974.

Madsen, I. C., Scarlett, N. V. Y. \& Kern, A. (2011). Z. Kristallogr. 226, 944-955.

Manceau, A., Chateigner, D. \& Gates, W. P. (1998). Phys. Chem. Miner. 25, 347-365.

McCusker, L. B., Von Dreele, R. B., Cox, D. E., Louër, D. \& Scardi, P. (1999). J. Appl. Cryst. 32, 36-50.

Scarlett, N. V. Y. \& Madsen, I. C. (2006). Powder Diffr. 21, 278-284.

Schmitt, B., Brönnimann, C., Eikenberry, E. F., Gozzo, F., Hörmann, C., Horisberger, R. \& Patterson, B. (2003). Nucl. Instrum. Methods Phys. Res. Sect. A, 501, 267-272.

Singh, B. \& Gilkes, R. J. (1992). Eur. J. Soil Sci. 43, 77-98.

Toby, B. H. (2006). Powder Diffr. 21, 67-70.

Tsipursky, S. I. \& Drits, V. A. (1984). Clay Miner. 19, 177-193.

Ufer, K., Roth, G., Kleeberg, R., Stanjek, H., Dohrmann, R. \& Bergmann, J. (2004). Z. Kristallogr. 219, 519-527.

Ufer, K., Stanjek, H., Roth, G., Dohrmann, R., Kleeberg, R. \& Kaufhold, S. (2008). Clays Clay Miner. 56, 272-282.

Wang, X., Li, J., Hart, R. D., van Riessen, A. \& McDonald, R. (2011). J. Appl. Cryst. 44, 902-910. 